\title{
Single residue (K332A) substitution in Kir6.2 abolishes the stimulatory effect of long-chain acyl-CoA esters: indications for a long-chain acyl-CoA ester binding motif
}

\author{
R. Bränström • I. B. Leibiger • B. Leibiger • \\ G. Klement • J. Nilsson • P. Århem • C. A. Aspinwall • \\ B. E. Corkey $\cdot$ O. Larsson $\cdot$ P.-O. Berggren
}

Received: 20 February 2007 / Accepted: 3 April 2007 / Published online: 24 May 2007

(C) Springer-Verlag 2007

\begin{abstract}
Aims/hypothesis The pancreatic beta cell ATP-sensitive potassium $\left(\mathrm{K}_{\text {ATP }}\right)$ channel, composed of the pore-forming $\alpha$ subunit Kir6.2, a member of the inward rectifier $\mathrm{K}+$ channel family, and the regulatory $\beta$ subunit sulfonylurea receptor 1 (SUR1), a member of the ATP-binding cassette superfamily, couples the metabolic state of the cell to electrical activity. Several endogenous compounds are known to modulate $\mathrm{K}_{\mathrm{ATP}}$ channel activity, including ATP, ADP, phosphatidylinositol diphosphates and long-chain acyl coenzyme A (LC-CoA) esters. LC-CoA esters have
\end{abstract}

R. Bränström · I. B. Leibiger · B. Leibiger · C. A. Aspinwall •

O. Larsson $\cdot$ P.-O. Berggren

Rolf Luft Research Center for Diabetes and Endocrinology,

Karolinska Institutet, Karolinska University Hospital,

Stockholm, Sweden

R. Bränström $(\bowtie)$

Section of Surgery, Department of Molecular Medicine and

Surgery, Karolinska Institutet,

Karolinska University Hospital P9:03,

17176 Stockholm, Sweden

e-mail: Robert.branstrom@ki.se

G. Klement · J. Nilsson • P. Århem

Nobel Institute for Neurophysiology,

Department of Neuroscience, Karolinska Institutet,

Stockholm, Sweden

O. Larsson

AstraZeneca R\&D,

Södertälje, Sweden

C. A. Aspinwall

Department of Chemistry, University of Arizona,

Tucson, AZ, USA

B. E. Corkey

Obesity Research Center, Boston University School of Medicine,

Boston, MA, USA been shown to interact with Kir6.2, but the mechanism and binding site(s) have yet to be identified.

Materials and methods Using multiple sequence alignment of known acyl-CoA ester interacting proteins, we were able to identify four conserved amino acid residues that could potentially serve as an acyl-CoA ester-binding motif. The motif was also recognised in the $\mathrm{C}$-terminal region of Kir6.2 (R311-332) but not in SUR1.

Results Oocytes expressing Kir6.2 $\Delta$ C26 K332A repeatedly generated $\mathrm{K}^{+}$currents in inside-out membrane patches that were sensitive to ATP, but were only weakly activated by $1 \mu \mathrm{mol} / 1$ palmitoyl-CoA ester. Compared with the control channel (Kir6.2 $\Delta$ C26), Kir6.2 $\Delta$ C26 K332A displayed unaltered ATP sensitivity but significantly decreased sensitivity to palmitoyl-CoA esters. Coexpression of Kir6.2 $\Delta$ C26 K332A and SUR1 revealed slightly increased activation by palmitoyl-CoA ester but significantly decreased activation by the acyl-CoA esters compared with the wild-type $\mathrm{K}_{\text {ATP }}$ channel and Kir6.2 $\Delta$ C26+SUR1. Computational modelling, using the crystal structure of KirBac1.1, suggested that K332 is located on the intracellular domain of Kir6.2 and is accessible to intracellular modulators such as LC-CoA esters.

Conclusions/interpretation These results verify that LCCoA esters interact at the pore-forming subunit Kir6.2, and on the basis of these data we propose an acyl-CoA ester binding motif located in the $\mathrm{C}$-terminal region.

Keywords Basic Science · Cells .

Beta cell signal transduction · Lipids/lipoproteins .

Other techniques

Abbreviations
$\begin{array}{ll}\text { LC-CoA } & \text { long-chain acyl coenzyme A } \\ \text { SUR } & \text { sulfonylurea receptor }\end{array}$

Abbreviations

SUR sulfonylurea receptor 


\section{Introduction}

In the pancreatic beta cell, the $\mathrm{K}_{\mathrm{ATP}}$ channel couples the metabolic state to electrical activity and insulin secretion. Briefly, closure of $\mathrm{K}_{\mathrm{ATP}}$ channels results in membrane depolarisation and the opening of voltage-gated $\mathrm{Ca}^{2+}$ channels, and thereby the triggering of insulin secretion. It is generally agreed that the regulation of the $\mathrm{K}_{\mathrm{ATP}}$ channel is mediated through the ATP/ADP ratio [1], but other endogenous signalling molecules, e.g. phosphatidylinositol4,5-diphosphate (PIP2) [2] and long-chain acyl coenzyme A (LC-CoA) esters [3], have been identified as modifiers of $\mathrm{K}_{\text {ATP }}$ channel activity.

Obesity is a major risk factor for the development of type 2 diabetes. Several studies have suggested that diets high in fat contribute to the development of the metabolic syndrome, obesity and type 2 diabetes. Parallel studies have shown a link between dietary fat composition and dysfunctional insulin secretion [4]. The mechanisms by which dietary fat may alter insulin secretion are not fully understood, partly because of the dual action of NEFA on insulin secretion. Short-term exposure to elevated NEFA levels stimulates insulin secretion, whereas long-term exposure to elevated NEFA levels decreases insulin secretion. One possible explanation could be intracellular accumulation of LC-CoA esters and thereby altered $\mathrm{K}_{\mathrm{ATP}}$ channel activity, maintaining the beta cell in a hyperpolarised, inactive state [3].

Despite the large number of estimated membrane-located $\mathrm{K}_{\text {ATP }}$ channels of the beta cell, only a small fraction (1-2\%) is thought to be active/open during resting periods [5]. Hence, only a small number of channels must be closed to trigger insulin secretion. Likewise, a small increase in channel activity hyperpolarises the beta cell plasma membrane and prevents exocytosis. Overactive $\mathrm{K}_{\text {ATP }}$ channels result in severe neonatal hyperglycaemia [6], which emphasises the central role of the channel. Endogenous $\mathrm{K}_{\mathrm{ATP}}$ channel activators, such as LC-CoA esters and PIP2, are therefore likely to be of considerable importance since the cellular content of ATP (low mmol/l) [7] causes nearly complete closure of the channel [1].

LC-CoA esters activate the pancreatic beta cell $\mathrm{K}_{\mathrm{ATP}}$ channels in all reported species and cell lines, including humans [8]. The esters do not require the presence of $\mathrm{Mg}^{2+}$ or ATP, and the site of action is separate from ADP [9]. Using the Kir6.2 $\Delta$ C26 variant, it has been shown that LC-CoA esters activate $\mathrm{K}^{+}$currents under conditions where sulfonylurea receptor 1 (SUR1) is not expressed $[10,11]$. However, since SUR1 does not function as a channel by itself, it remains to be investigated whether or not LC-CoA esters also bind to SUR1. Kir6.2 is inhibited by ATP and opened by PIP2 and LC-CoA esters. Mutation of lysine-185 has been shown to abolish the inhibitory effect of ATP. The interaction of PIP2 is thought to be linked mainly to arginine 54 [12], whereas the involvement of specific amino acid residues of Kir6.2 in the modulation by LC-CoA esters has not been demonstrated.

Pancreatic beta cell $\mathrm{K}_{\text {ATP }}$ channels consist of four poreforming $\alpha$ subunits (Kir6.2) and four regulatory $\beta$ subunits (SUR1), and both types of subunit are required for a fully functional $\mathrm{K}_{\mathrm{ATP}}$ channel $[13,14]$. Regulatory compounds act through both $\alpha$ and $\beta$ subunits, whereas the inhibitory effect of sulfonylurea and the activating effect of ADP are confined to the $\beta$ subunit [15]. Conversely, the inhibitory effect of ATP and the activating effect of PIP2 and LC-CoA esters are localised to the Kir6.2 $[10,11,16]$. In this study we used site-directed mutations of the truncated version of Kir6.2 (Kir6.2 $\Delta$ C26) to investigate the modulation of Kir6.2 by LC-CoA esters.

\section{Materials and methods}

Animals and preparation of cells Electrophysiological recordings from native $\mathrm{K}_{\mathrm{ATP}}$ channels were performed using excised membrane patches from adult ob/ob (Lep/ Lep) mouse pancreatic beta cells. In brief, the $o b / o b$ mice were obtained from a local colony [17] and fasted for $24 \mathrm{~h}$ before decapitation. Islets were isolated by a collagenase (Roche, Basel, Switzerland) technique described by Lacy and Kostianovsky [18].

Xenopus oocytes were collected from extra-large Xenopus laevis females. The animals were anaesthetised with 3-aminobenzoic acid methyl ester (Sigma, St Louis, MO, USA; $1.5 \mathrm{~g} / 1$ of water). Oocytes were removed via a small bowel incision and oocytes were defolliculated using a collagenase A method described elsewhere [11]. Oocytes, stage $\mathrm{V}-\mathrm{VI}$, were injected with $0.5-5 \mathrm{ng}$ of mRNA $/ 50 \mathrm{nl}$ sterile RNase-free water encoding Kir6.2 $\Delta$ C26, Kir6.2 $\Delta$ C26 W311A, Kir6.2 $\Delta$ C26 D329A, Kir6.2 $\Delta$ C26 K332A, Kir6.2AC26 W311A K332A and Kir6.2 $\Delta$ C26 W311A D329A K332A. SUR1 was only coexpressed with functional Kir6.2 $\Delta \mathrm{C} 26$ mutant clones. Oocytes were maintained at $19^{\circ} \mathrm{C}$ for $2-5$ days before use. All animal studies were approved by the local ethics committee of the Karolinska University Hospital.

Vector construction The generation of plasmids $\mathrm{pB}$. mKir6.2 $\Delta \mathrm{C} 26$ and pB.SUR1 has been described previously [11]. Plasmids for the pB.mKir6.2 $\Delta \mathrm{C} 26$ mutants K332A, W311A and D329A were generated by site-directed mutagenesis using the QuikChange Mutagenesis Kit (Stratagene, La Jolla, CA, USA) and oligonucleotides were purchased from Proligo (Paris, France), bearing the following nucleotide changes: W311A (TGG $\rightarrow$ GCA); D329A $(\mathrm{GAC} \rightarrow \mathrm{GCC})$; K332A (AAA $\rightarrow \mathrm{GCA})$. In all plasmids the 
orientation of the expression cassette was chosen to allow transcripts to be obtained with T7 RNA polymerase. All vector constructs were verified by DNA sequence analysis.

In vitro transcription Plasmid DNA was prepared using a QIAprep Spin Miniprep Kit (Qiagen, Hilden, Germany) and purified using a GenePrep Kit (Ambion, Austin, TX, USA). The respective plasmid DNA was linearised by digestion with $X b a \mathrm{I}$, purified by phenol-chloroform treatment, and ethanolprecipitated. The DNA pellet was redissolved in water and an aliquot containing $0.5-1 \mu \mathrm{g}$ DNA was used for in vitro transcription. Capped mRNA was synthesised by employing the mMessenger mMachine T7 Kit (Ambion). The purified mRNA was dissolved in $10 \mathrm{mmol} / \mathrm{l}$ Tris- $\mathrm{HCl}(\mathrm{pH} 7.4)$ and stored in aliquots at $-80^{\circ} \mathrm{C}$.

Electrophysiology Potassium channel activity was recorded using the patch-clamp technique [19] with an Axopatch 200B patch-clamp amplifier (Axon Instruments, Foster City, CA, USA). The recorded signal was low-passfiltered at $2 \mathrm{kHz}$ and stored on magnetic tape (JVC, Tokyo, Japan). Single-channel current traces were displayed according to the prevailing convention, upward deflection denoting outward currents. All experiments were performed at room temperature (approximately $22^{\circ} \mathrm{C}$ ) and channel activity was measured at a membrane potential of $0 \mathrm{mV}$. The standard extracellular solution contained (in $\mathrm{mmol} / \mathrm{l}$ ) $138 \mathrm{NaCl}, 5.6 \mathrm{KCl}, 1.2 \mathrm{MgCl}_{2}, 2.6 \mathrm{CaCl}_{2}$ and 5 HEPES$\mathrm{NaOH}$ at $\mathrm{pH}$ 7.4. For inside-out recordings, the intracellular-like solution (i.e. bath solution) consisted of (in mmol/l) $125 \mathrm{KCl}, 1 \mathrm{MgCl}_{2}, 10$ EGTA, $25 \mathrm{KOH}$ and 5 HEPES-KOH at $\mathrm{pH}$ 7.15. ATP and palmitoyl-CoA ester (C16:0) were added to the intracellular solution at the final concentration indicated in the text and figures. Palmitoyl-CoA (C16:0) was prepared as a stock solution in deionised water (Millipore, Billerica, MA, USA), and ATP was added as $\mathrm{Mg}$ salt. All chemicals were of analytical grade and were obtained from Sigma.

Data analysis For the mean current analysis, channel recordings were filtered at $0.2 \mathrm{kHz}(-3 \mathrm{~dB}$ value, eightpole Bessel filter; Frequency Devices, Haverhill, MA, USA), digitised at $0.8 \mathrm{kHz}$ and stored in a computer, using an analogue-digital converter (TL-1; Axon Instruments). The mean current was calculated as previously described [20]. Data are presented as mean \pm SD. For the analysis of single-channel kinetics, 30-s segments of channel recordings were filtered at $1 \mathrm{kHz}$ and digitised at $5 \mathrm{kHz}$. Calculations were performed using TAC software (Bruxton, Seattle, WA, USA). The analysis of kinetic parameters was restricted to segments of the experimental recordings containing a maximum of three active channels. Channel activity was compared using Student's $t$ test or ANOVA for multiple groups; $p$ values less than 0.05 were considered significant. All experiments were repeated at least three times with similar results unless stated otherwise.

\section{Results}

Multiple sequence alignment LC-CoA esters bind and potently affect many different intracellular enzymes and proteins (see review by Shrago [21]), though the binding motif(s) for LC-CoA ester in these proteins is unknown. To identify potential amino acid residues involved in acyl-CoA ester binding, we used multiple amino acid sequence alignments of proteins that bind or are affected by LCCoA esters. Figure 1a shows several LC-CoA ester binding proteins that were used in a multiple sequence alignment procedure employing the protein-protein BLAST software (NCBI, NLM, NIH, Bethesda, MD, USA). Multiple amino acid sequence alignments of these proteins revealed an amino acid stretch containing four conserved amino acid residues. Initially, we identified a conserved valine $(\mathrm{V})$, aspartic acid (D) and lysine (K). We then extended the binding motif to include a conserved aromatic amino acid group of $10-15$ proximal amino acids, i.e. in the N-terminal direction (W, F or Y). Since LC-CoA ester has been shown to interact with the $\alpha$ subunit of the $\mathrm{K}_{\mathrm{ATP}}$ channel, namely Kir6.2 [10, 11], we performed a sequence alignment between our proposed LC-CoA ester binding motif and Kir6.2 (Fig. 1b). A proposed LC-CoA ester binding motif could be identified in the C-terminal region of Kir6.2, between residues 311 and 332. The same region was highly conserved in the mouse, rat and human (data not shown). PIP2 has been shown to activate all Kir channels, whereas activation by LC-CoA esters seems to be restricted to Kir6.2. However, sequence alignment of the Kir superfamily shows that Kir2.1, Kir3.4 and Kir6.1 do indeed have a potential LC-CoA ester binding motif (Fig. 1c), though, interestingly, Kir2.1 and Kir3.4 were shown not to be activated but rather inhibited by LC-CoA esters [10, 22]. Thus, it is possible that LC-CoA ester binding is linked to a blocking mechanism within the channel protein as well. No significant sequence match was identified in Kir7.1 or SUR1.

Mutation of amino acids As stated above, we identified four conserved amino acid residues, as indicated in Fig. 1. To test the involvement of these amino acids in Kir6.2 modulation by a LC-CoA ester motif, we introduced pointdirected mutations in Kir6.2 $\Delta$ C26, i.e. single or multiple amino acid exchanges (Table 1). In Xenopus oocytes expressing Kir6.2 $\Delta$ C26 W311A and Kir6.2 $\Delta$ C26 D329A, no current could be detected in single-channel recordings 
a

\begin{tabular}{|c|c|c|c|}
\hline h_LCL1 & 533 & DGWLHT .G. . . D I GKWL PNGT LK I I DRKK & 557 \\
\hline h_ACBP & 57 & DAWNELKG . . TS KEDAMKAY I NKVEEL & 83 \\
\hline h_ANT1 & 79 & I RYF PTQALNFAF KDKYKQLF LGGVDR HK & 107 \\
\hline h_LCEH & 541 & DGPGFYTTRCLA. PMMSEV I R I LQEGVDPKK & 570 \\
\hline h_UP3 & 219 & CHFVSAFG.....AGFCATVVAS PVDVV & 242 \\
\hline h_ACTH & 331 & TYVSLSQE . . GR SLPVPQLVPETEDEKK & 356 \\
\hline h_ACD & 313 & YRWH INFT . . TF F I DCMAALGLAYDRKI & 338 \\
\hline
\end{tabular}

b

309 I LWGQRFVP I VAEEDGRYSVDYSK 332

h_Kir6.2

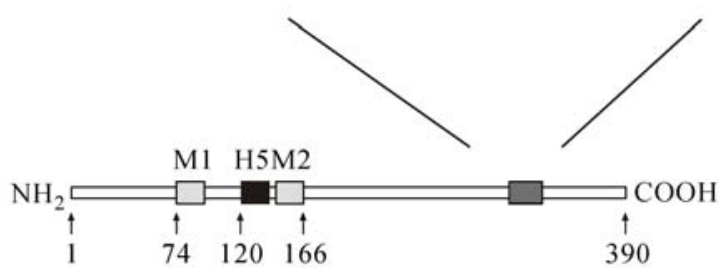

C

$\begin{array}{lllllll}\text { h_Kir6.2 } & 309 & \text { ILWGQRFVPIVAEEDGRYSVDYSK } & 332 & \text { X } & \text { Non Conserved } \\ \text { h_Kir6.1 } & 317 & \text { IQWGHRFVSIVTEEEGVYSVDYSK } & 340 & & \text { Similar } \\ \text { h_Kir3.4 } & 327 & \text { VLWGHRFTPVLTLEKGFYEVDYNT } & 350 & \text { X } & \text { Conserved } \\ \text { h_Kir2.1 } & 320 & \text { ILWGHRYEPVLFEEKHYYKVDYSR } & 343 & \text { X } & \text { All match }\end{array}$

Fig. 1 a Multiple sequence alignment of various proteins that interact with LC-CoA esters. $h \_L C L$ human long-chain fatty acid CoA ligase, $\mathrm{P} 33121 ; h$ ACBP human acyl-CoA binding protein, $\mathrm{P} 07108 ; h$ ANT1 human adenine nucleotide translocase 1 , protein access code P12235; $h \_$LCEH human long chain enoyl-CoA hydratase, protein access code P40939; $h$ _UP3 human uncoupling protein 3, protein access code

from membrane patches using the inside-out configuration, in agreement with a previous report [23]. Replacing the lysine (K) with an alanine (A) at position 332 (K332A) resulted in a basal $\mathrm{K}^{+}$current at the same intensity as Kir6.2 $\Delta$ C26 (Table 1). Although channel currents were obtained using Kir6.2 $\Delta$ C26 mutants, wild-type $\mathrm{K}_{\mathrm{ATP}}$ channels are composed of both Kir6.2 and SUR1 subunits. Coexpression of Kir6.2 $\Delta$ C26 with SUR1 results in in-
P55916; $h$ ACTH human acyl CoA thioester hydrolase, protein access code O00154; $h_{-} A C D$ human acyl-CoA desaturase, protein access code O00767. b A similar signature sequence was found in the Kir6.2 C-terminal region. M1 and M2 represent transmembrane regions; H5 represents a pore-forming loop. c Several Kir channels share sequence homology with the proposed LC-CoA ester-binding motif

creased basal current and increased sensitivity to ATP [24]. Additionally, several regulatory compounds, e.g. sulfonylurea, diazoxide and ADP, are known to interact with the SUR1 subunit. Therefore, we coexpressed the functional Kir6.2 clones generated herein (Kir6.2 $\Delta \mathrm{C} 26$ and Kir6.2 $\Delta$ C26 K332A) with SUR1. The resulting basal currents were increased by a factor of approximately 3 compared with $\operatorname{Kir} \Delta \mathrm{C} 26$ clones alone (Table 1).
Table 1 Mutations directed at a proposed LC-CoA ester binding site in Kir6.2

The $95 \%$ CI of the basal current is presented for freshly excised inside-out patches expressing Kir6.2 $\Delta$ C26 clones, either alone or together with SUR1. ${ }^{\mathrm{a}} p=\mathrm{NS}$ vs Kir6.2 $\Delta \mathrm{C} 26$ ${ }^{\mathrm{b}} p=\mathrm{NS}$ vs Kir6.2 $\Delta \mathrm{C} 26+\mathrm{SUR} 1$

\begin{tabular}{lll}
\hline$\alpha$ Subunit & $\beta$ Subunit & Basal current $(95 \% \mathrm{CI})$ \\
\hline Kir6.2 $\Delta$ C26 & - & $4.1-16.8 \mathrm{pA}(n=6)$ \\
Kir6.2 $\Delta$ C26 W311A & - & No current $^{\mathrm{a}}$ \\
Kir6.2 $\Delta$ C26 D329A & - & No current $^{\mathrm{a}}$ \\
Kir6.2 $\Delta$ C26 K332A & - & $7.5-21.8 \mathrm{pA}(n=11)^{\mathrm{a}}$ \\
Kir6.2 $\Delta$ C26 W311A K332A & - & No current \\
Kir6.2 $\Delta$ C26 W311A D329A K332A & - & No current \\
Kir6.2 $\Delta$ C26 & SUR1 & $14.6-24.0 \mathrm{pA}(n=3)$ \\
Kir6.2 $\Delta$ C26 K332A & SUR1 & $16.4-63.8 \mathrm{pA}(n=5)^{\mathrm{b}}$
\end{tabular}


a

$1 \mu \mathrm{mol} / 1$ Palmitoyl-CoA (C16:0)
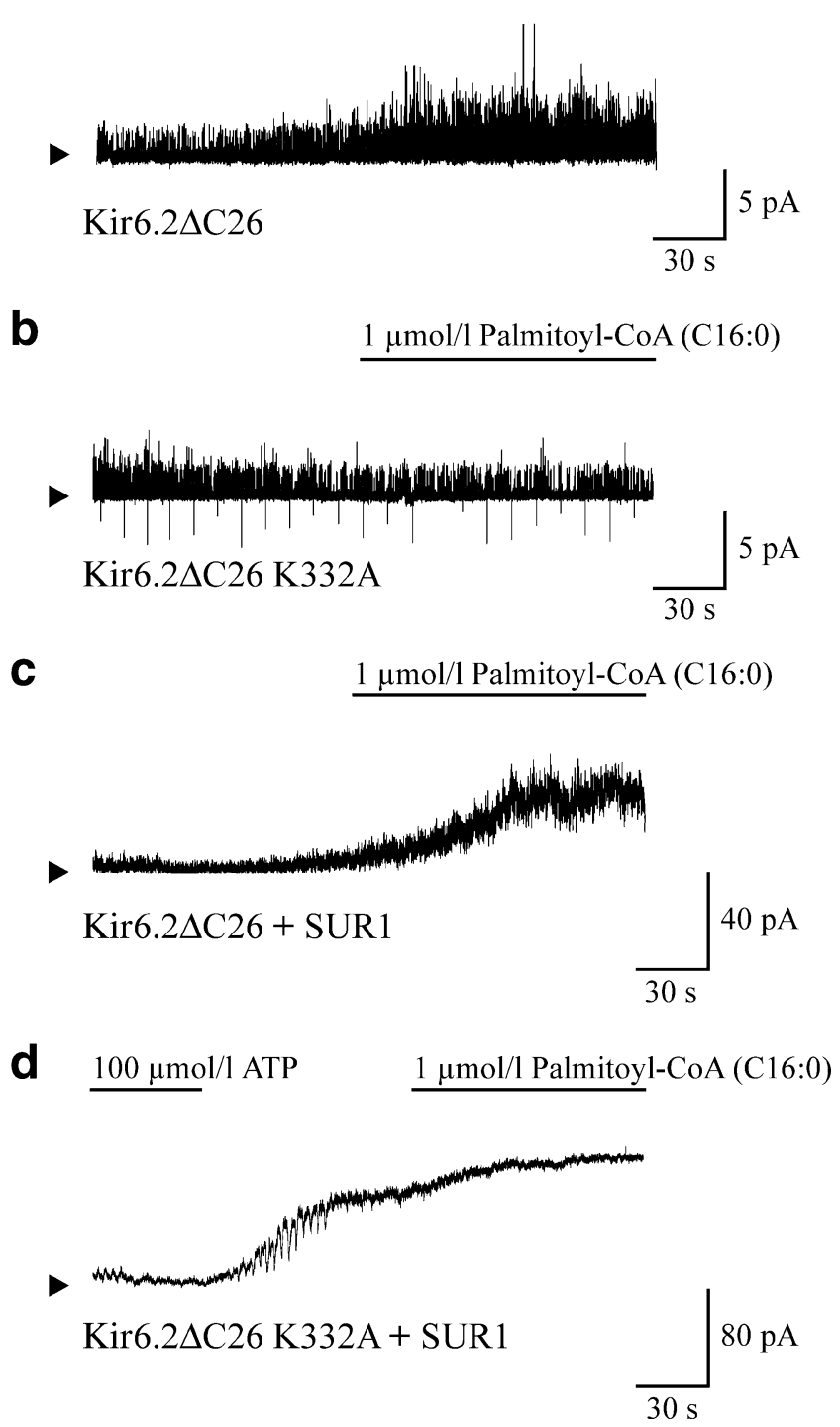

Fig. 2 Effects of palmitoyl-CoA esters on excised membrane patches. In oocytes expressing Kir6.2 $\Delta \mathrm{C} 26$, addition of $1 \mu \mathrm{mol} / \mathrm{l}$ palmitoylCoA ester activated the channel (a), whereas oocytes expressing Kir6.2 $\Delta$ C26 K332A were not affected by the addition of palmitoylCoA ester (b). c, d Oocytes were injected with the same Kir clones as in $\mathbf{a}$ and $\mathbf{b}$, together with SUR1. $\mathrm{K}^{+}$currents in oocytes expressing Kir6.2 $\Delta$ C26+SUR1 increased approximately fivefold in the presence of $1 \mu \mathrm{mol} / 1$ palmitoyl-CoA ester (c). Kir6.2 $\Delta$ C26 K332A+SUR1 currents were only slightly increased in the presence of $1 \mu \mathrm{mol} /$ 1 palmitoyl-CoA ester (d). The arrowhead represents zero current

Channel currents in excised membrane patches Figure 2 illustrates the effect of palmitoyl-CoA ester $(1 \mu \mathrm{mol} / \mathrm{l})$ on freshly isolated membrane patches from oocytes expressing Kir6.2 $\Delta$ C26, Kir6.2 $\Delta$ C26 K332A, Kir6.2 $\Delta$ C26+SUR1 and Kir6.2 $\Delta$ C26 K332A+SUR1. Kir6.2 $\Delta$ C26 was activated by palmitoyl-CoA ester, and the mean current increased from $2.3 \pm 0.7$ to $6.0 \pm 1.3 \mathrm{pA}$ (Fig. $2 \mathrm{a}, 290 \pm 40 \%, n=6 ; p<$ 0.01 ), an increase of similar magnitude to that reported in a

$0.1 \mathrm{mmol} / 1$ Tolbutamide

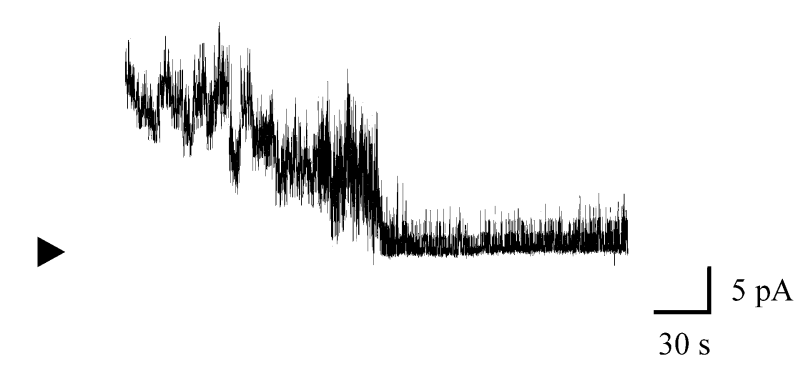

Kir6.2AC26 K332A + SUR1

b

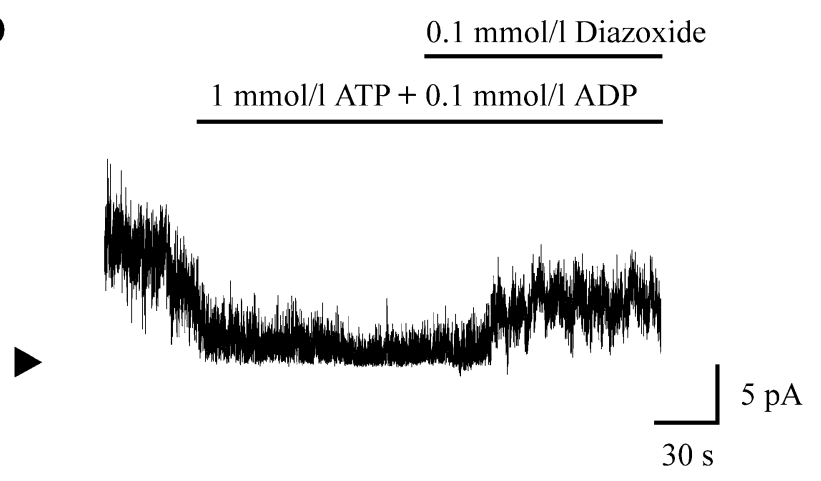

Kir6.2 $\Delta$ C26 K332A + SUR1

Fig. 3 Effects of tolbutamide and diazoxide on excised membrane patches from oocytes injected with Kir6.2 $\Delta$ C26 K332A+SUR1. a The mean current decreased from 16.1 to $0.8 \mathrm{pA}$ after addition of $0.1 \mathrm{mmol} / 1$ tolbutamide. $\mathbf{b}$ The addition of ATP/ADP at a ratio of $10: 1$ decreased the current from 7.2 to $1.1 \mathrm{pA}$. Inclusion of $0.1 \mathrm{mmol} /$ 1 diazoxide increased the current to $3.7 \mathrm{pA}$. The arrowhead represents zero current

native pancreatic beta cell $\mathrm{K}_{\mathrm{ATP}}$ channels [3]. Conversely, single-residue mutation of 332, from lysine $(\mathrm{K})$ to alanine (A), did not increase channel current upon exposure to palmitoyl-CoA ester (Fig. 2b). In 11 excised inside-out membrane patches, mean current was estimated to be $2.6 \pm$ $1.0 \mathrm{pA}$ under control conditions, increasing to $3.4 \pm 1.6 \mathrm{pA}$ (110 $\pm 8 \%$, paired comparison; NS) upon exposure to $1 \mu \mathrm{mol} / 1$ palmitoyl-CoA ester. A detailed analysis of channel kinetics showed a mean open time of $8.1 \pm 4.3 \mathrm{~ms}$ for Kir6.2 $\Delta$ C26 K332A under control conditions, similar to the mean open time reported for Kir6.2 $\Delta$ C26 [11]. In the presence of palmitoyl-CoA ester, the mean open time of Kir6.2 $\Delta$ C26 K332A was $7.7 \pm 3.8$ ms (NS), which should be compared with the almost threefold increase seen for Kir6.2 $\Delta$ C26 [11]. To verify channel function, ATP was applied to each membrane patch before exposure to the ester. In the Kir6.2 $\Delta \mathrm{C} 26 \mathrm{~K} 332 \mathrm{~A}$ channels, addition of $1 \mathrm{mmol} / 1$ ATP resulted in a decrease in channel current from $1.5 \pm 0.4$ to $0.2 \pm 0.1 \mathrm{pA}(p<0.01)$. Single-residue mutation of W311 and D329 did not result in any $\mathrm{K}^{+}$current in isolated membrane patches, nor did any combination of 

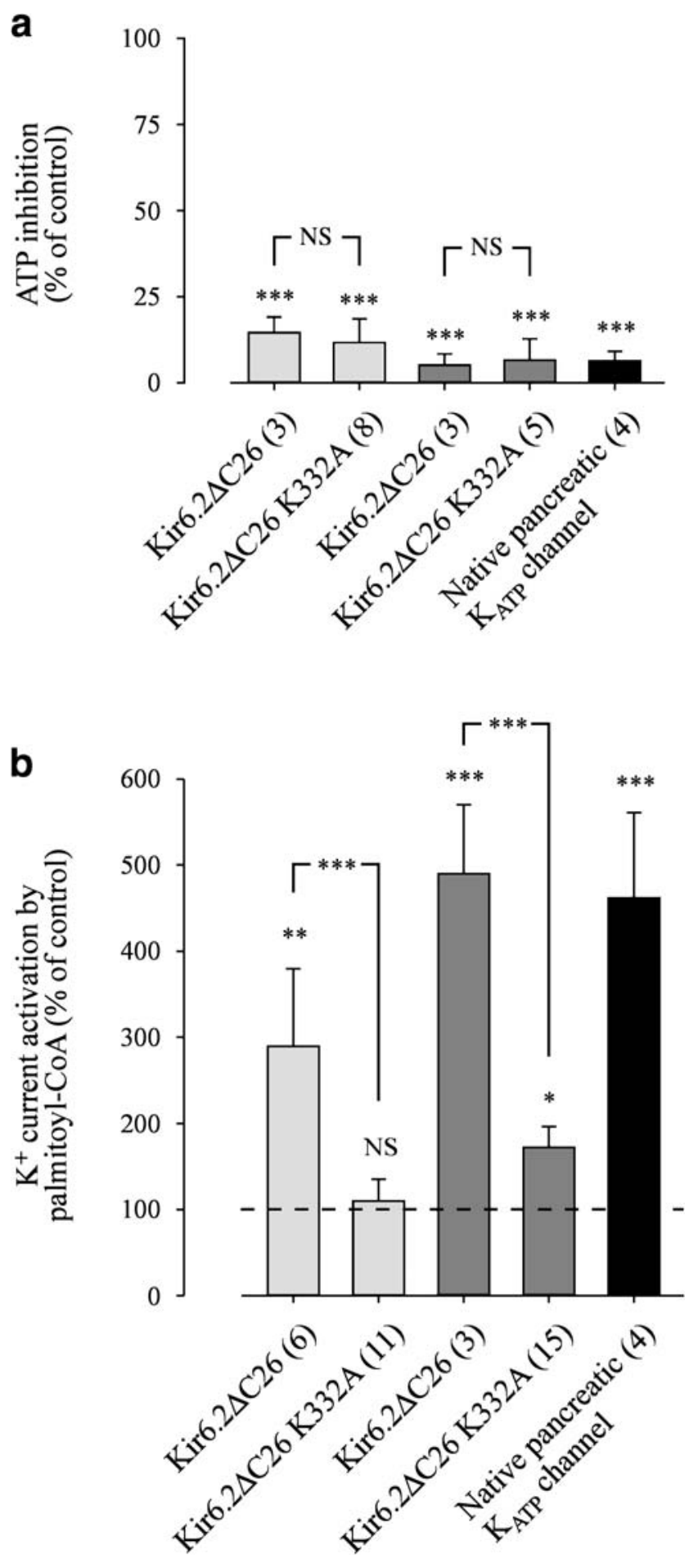

these mutations result in $\mathrm{K}^{+}$current (Table 1), even after application of $1 \mu \mathrm{mol} / \mathrm{l}$ palmitoyl-CoA ester (data not shown).

Coexpression of Kir6.2 $\Delta \mathrm{C} 26$ and SUR1 resulted in a channel current with properties close to those of the wildtype $\mathrm{K}_{\text {ATP }}$ channel (Kir6.2/SUR1) [24]. Exposing patches excised from oocytes expressing Kir6.2 $\Delta \mathrm{C} 26$ and SUR1 to ATP $(100 \mu \mathrm{mol} / \mathrm{l})$ decreased channel current from $16 \pm 4$ to $0.1 \pm 0.2$ pA (Fig. 2 c $, n=3, p<0.001$ ). Addition of $1 \mu \mathrm{mol} /$ 1 palmitoyl-CoA ester activated the channel and the mean current increased to $91 \pm 7.6 \mathrm{pA}$, compared with $19 \pm 2.2 \mathrm{pA}$ in the control situation $(p<0.001)$. Functional mutated Kir6.2 $\Delta$ C26 clones (i.e. K332A) were also coexpressed with SUR1. Membrane recordings from Kir6.2 $\Delta \mathrm{C} 26$ K332A+SUR1 displayed normal ATP sensitivity, with channel current decreasing from $40.1 \pm 13.5$ to $0.2 \pm$ $0.12 \mathrm{pA}$ (Fig. 2d, $n=5, p<0.001$ ). Inclusion of $1 \mu \mathrm{mol} /$ 1 palmitoyl-CoA ester increased channel current from $22.1 \pm$ 7 to $38 \pm 12 \mathrm{pA}(p<0.05)$. Activation by palmitoyl-CoA ester was significantly decreased in Kir6.2 $\Delta$ C26 K332A +SUR1 compared with Kir6.2 $\Delta$ C26+SUR1 $(p<0.001)$ (Fig. 4). Other known agonists and antagonists acting through SUR1, such as ADP, diazoxide and tolbutamide, displayed potencies similar to those of the $\mathrm{K}_{\mathrm{ATP}}$ wild-type channel (Fig. 3). Compiled data on the effect of ATP and palmitoyl-CoA ester on Kir variants, with and without the presence of SUR1 and the native pancreatic beta cell $\mathrm{K}_{\mathrm{ATP}}$ channel are shown in Fig. 4. No difference was observed between Kir6.2 $\Delta \mathrm{C} 26$ and Kir6.2 $\Delta$ C26 K332A with regard to ATP sensitivity. When SUR1 was coexpressed with Kir variants, ATP sensitivity increased dramatically, but remained unaltered compared with native $\mathrm{K}_{\mathrm{ATP}}$ channels. When Kir variants were exposed to palmitoyl-CoA (1 $\mu \mathrm{mol} / 1$ ), Kir6.2 $\Delta$ C26 K332A channels were not significantly activated compared with Kir6.2 $\Delta$ C26 channels. Coexpression of SUR1 with Kir6.2AC26 K332A did not restore the sensitivity to palmitoyl-CoA.

\section{Discussion}

The $\mathrm{K}_{\text {ATP }}$ channel plays a critical role in stimulus-secretion coupling in the pancreatic beta cell, where it couples the metabolic state to electrical activity and insulin secretion.

Fig. 4 Compiled data on the effect of ATP and palmitoyl-CoA esters on Kir6.2 $\Delta$ C26 and Kir6.2 $\Delta$ C26 K332A, with and without SUR1, and the native pancreatic $\mathrm{K}_{\mathrm{ATP}}$ channel. a When Kir clones were expressed without SUR1 (light grey bars) the ATP concentration was $1 \mathrm{mmol} / \mathrm{l}$, whereas in patches isolated from oocytes co-injected with SUR1 (dark grey bars), $0.1 \mathrm{mmol} / 1$ ATP was used. $\mathbf{b}^{+}$current is expressed as a percentage of $\mathrm{K}^{+}$current recorded before the addition of $1 \mu \mathrm{mol} / 1$ palmitoyl-CoA (C16:0). Number of experiments is given in parentheses. ${ }^{*} p<0.05, * * p<0.01, * * * p<0.001$ vs channel activity before the addition of palmitoyl-CoA ester Even though the ATP/ADP ratio has been proposed as the major regulator of $\mathrm{K}_{\mathrm{ATP}}$ channel activity in the intact beta cell under normal conditions [25], other activators, such as LC-CoA esters, may well have importance under pathophysiological conditions. In situations associated with increased levels of circulating NEFA, LC-CoA esters have been shown to accumulate within cells and to decrease insulin secretion [3]. 


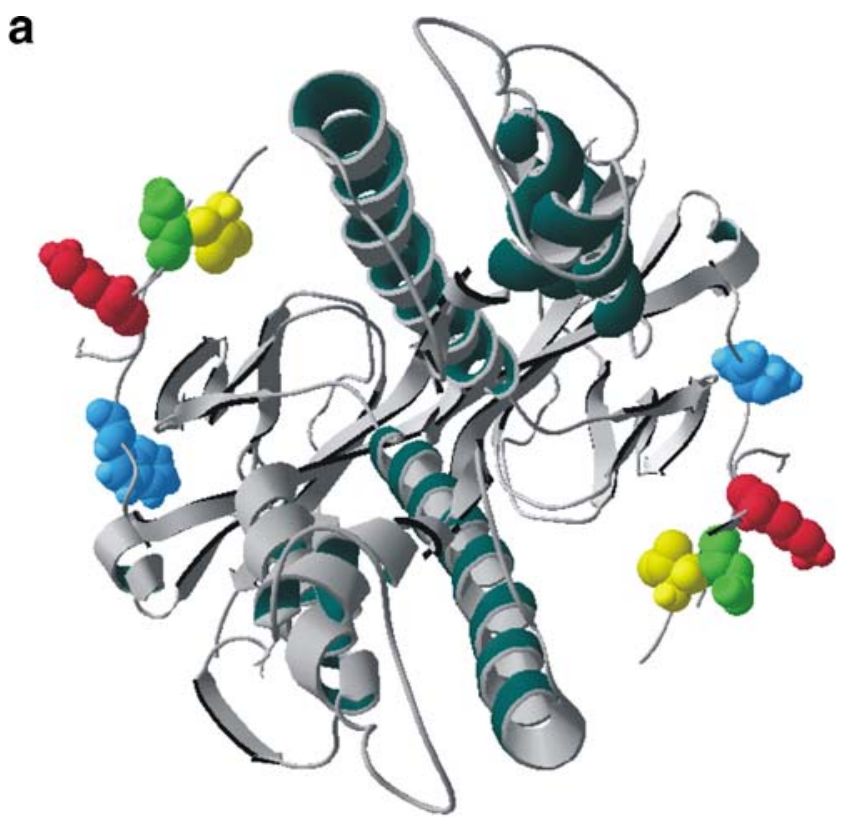

b

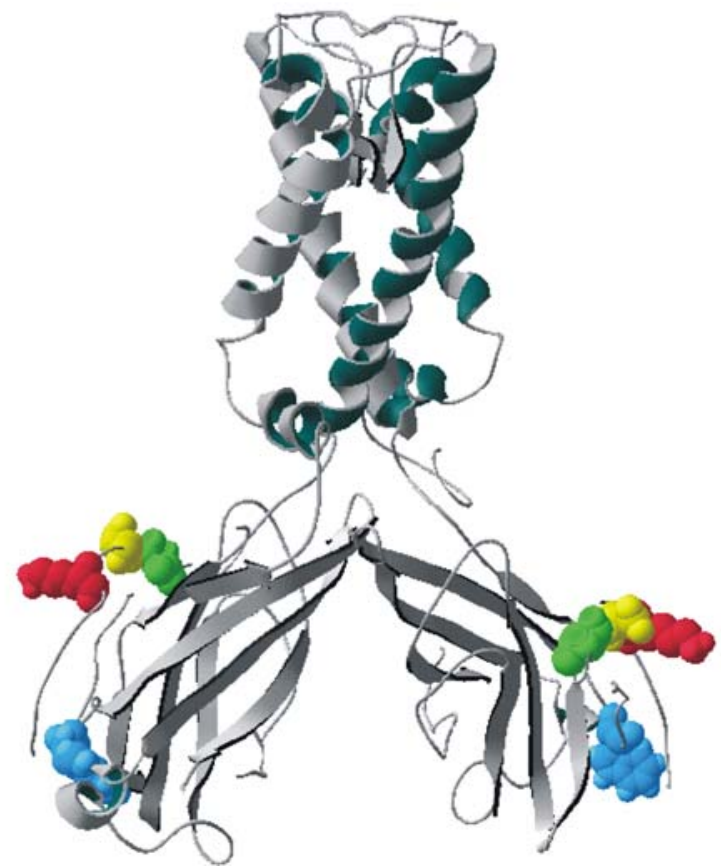

Fig. 5 Three-dimensional structure of two KirBac1.1 subunits (accession code 1P7B; RCSB Protein Data Bank) with mark residues W281 (blue), I298V (green), D299 (yellow) and R302K (red) to show the tentative location of the proposed LC-CoA ester binding motif in Kir6.2. a Top view. b Side view. Identification of residues W281, I298, D299 and R302 as corresponding to residues W311, V328, D329 and K332 in Kir6.2 was based on the alignment of KirBac1.1 and human Kir6.2 (Q14564), using SDSC Biology WorkBench version 3.2. The three-dimensional structure was obtained by the use of Swiss-Pdb viewer, version 3.7 (fixed selected side change)

Oocytes expressing the truncated version of Kir6.2, i.e. $\Delta \mathrm{C} 26$, have been shown to be activated by LC-CoA esters $[10,11]$. In this study we identified conserved residues in known LC-CoA ester binding proteins using multiple sequence alignments, suggesting a potential LC-CoA binding motif. We found a conserved region containing a fouramino-acid signature (W/F/Y...VD..K) among proteins known to bind LC-CoA esters. At present the crystal structure of four different acyl CoA ester binding proteins are known. Even though the crystal structure of these proteins does not give an exact location or signature of an acyl-CoA ester binding site/motif, it has been proposed that positive residues, such as lysine $(\mathrm{K})$, and tryptophan $(\mathrm{W})$ are involved in the binding of LC-CoA ester [26]. The proposed motif was also identified in the C-terminal stretch of Kir6.2. We modified these amino acids within Kir6.2 $\Delta$ C26 by sitedirected mutations to obtain the Kir6.2 $\Delta \mathrm{C} 26$ variants W311A, D329A and K332A. Mutation of K332 resulted in a Kir6.2 $\Delta$ C26 current that was not activated by LC-CoA esters, but displayed a normal regulatory pattern with regard to ATP, ADP, diazoxide and sulfonylurea when coexpressed with SUR1. To get information on the location of the identified residues in the three-dimensional structure of the Kir6.2 channel, we used computational modelling. We introduced a lysine at location 302 of the crystal structure of the inward rectifier potassium channel KirBac1.1 (closed) from the eubacterium Burkholderia pseudomallei, the only Kir channel so far crystallised and structurally determined [27]. Kir1.1 has approximately $45 \%$ sequence identity with Kir6.2. Location R302 in KirBac1.1 was identified as corresponding to location K332 in Kir6.2 by aligning the two channel sequences (see legend of Fig. 5). Figure 5 shows that 281-302 is located in the cytoplasmic domain of the channel protein facing the cytosol, suggesting that the residue is accessible to intracellular modulators such as LCCoA esters.

It has been proposed that PIP2 and LC-CoA esters share the same regulatory mechanism, and possibly also interact at a common binding site [28]. However, the proposed LCCoA ester binding motif, which we have now identified as 311-332, is not close to the residues that have been proposed for ATP and PIP2 interaction [12, 17, 24, 29, 30], except for 314 [31] and 308-311 [23]. All members of the Kir channel family tested so far are activated by PIPs, especially PIP2. In contrast, LC-CoA esters appear to be specific modulators of $\mathrm{K}_{\mathrm{ATP}}$ channels since none of the other Kir channels (Kir1.1, Kir2.1, Kir3.4, Kir4.1 and Kir7.1) are activated [22]. Scanning through the Kir family for a proposed LC-CoA ester binding motif reveals a region close to the C-terminal in Kir2.1 and Kir3.4 with high homology to our proposed acyl-CoA ester binding motif. Interestingly, mutated Kir2.1 channels that are made insensitive to PIPs are activated by LC-CoA esters [32]. It is attractive to speculate that both Kir2.1 and Kir3.4 do indeed possess an LC-CoA ester binding motif (Fig. 1d), but that the stimulatory effect is masked by a mechanism that remains to be defined. However, it is noteworthy that in 
Kir7.1 no consensus sequence was found, yet this channel is inhibited by LC-CoA esters [21].

We conclude that LC-CoA esters interact with Kir6.2, and not with SUR1. This notion is based on the finding that a single amino acid mutation in Kir6.2 $\Delta$ C26 abolishes the stimulatory effect of LC-CoA esters, which is not restored by coexpression with SUR1. We also propose an LC-CoA ester binding motif in Kir6.2, and conclude that this may be a useful tool for exploring the role of LC-CoA esters in pancreatic beta cell physiology and pathophysiology.

Acknowledgements This work was supported by grants from the Swedish Research Council, the Swedish Diabetes Association, the Swedish Society of Medical Research, the Nordic Insulin Foundation Committee, Novo Nordisk Foundation, Funds of the Karolinska Institutet, Jeanssons Foundation, Åke Wiberg's Foundation, Thurings Foundation, Tore Nilsson's Foundation, Berth von Kantzow's Foundation, The Family Stefan Person Foundation and NIH grant RO1-DK35914.

Duality of interest This work involved no duality of interest.

\section{References}

1. Ashcroft FM, Rorsman P (1989) Electrophysiology of the pancreatic beta-cell. Prog Biophys Mol Biol 54:87-143

2. Hilgemann DW, Ball R (1996) Regulation of cardiac $\mathrm{Na}+$, $\mathrm{Ca} 2+$ exchange and KATP potassium channels by PIP2. Science 273:956-959

3. Larsson $\mathrm{O}$, Deeney JT, Bränström R, Berggren PO, Corkey BE (1996) Activation of the ATP-sensitive $\mathrm{K}+$ channel by long chain acyl-CoA. A role in modulation of pancreatic beta-cell glucose sensitivity. J Biol Chem 271:10623-10626

4. Salmeron J, Hu FB, Manson JE, Stampfer MJ, Colditz GA, Rimm EB, Willett WC (2001) Dietary fat intake and risk of type 2 diabetes in women. Am J Clin Nutr 73:1019-1026

5. Cook DL, Satin LS, Ashford ML, Hales CN (1988) ATP-sensitive $\mathrm{K}+$ channels in pancreatic beta-cells. Spare-channel hypothesis. Diabetes 37:495-498

6. Koster JC, Marshall BA, Ensor N, Corbett JA, Nichols CG (2000) Targeted overactivity of beta cell K(ATP) channels induces profound neonatal diabetes. Cell 100:645-654

7. Tamarit-Rodriquez J, Hellman B, Sehilin J (1977) Metabolic characteristics of pancreatic beta-cells exposed to calcium-transporting ionophores. Biochim Biophys Acta 496:167-174

8. Bränström R, Aspinwall CA, Välimäki S et al (2004) Long-chain CoA esters activate human pancreatic beta-cell KATP channels: potential role in type 2 diabetes. Diabetologia 47:277-283

9. Bränström R, Corkey BE, Berggren PO, Larsson O (1997) Evidence for a unique long chain acyl-CoA ester binding site on the ATP-regulated potassium channel in mouse pancreatic beta cells. J Biol Chem 272:17390-17394

10. Gribble FM, Proks P, Corkey BE, Ashcroft FM (1998) Mechanism of cloned ATP-sensitive potassium channel activation by oleoyl-CoA. J Biol Chem 273:26383-26387

11. Bränström R, Leibiger IB, Leibiger B, Corkey BE, Berggren PO, Larsson O (1998) Long chain coenzyme A esters activate the pore-forming subunit (Kir6.2) of the ATP-regulated potassium channel. J Biol Chem 273:31395-31400

12. Schulze D, Krauter T, Fritzenschaft H, Soom M, Baukrowitz T (2003) Phosphatidylinositol 4,5-bisphosphate (PIP2) modulation of ATP and pH sensitivity in Kir channels. A tale of an active and a silent PIP2 site in the $\mathrm{N}$ terminus. J Biol Chem 278:10500-10505

13. Inagaki N, Gonoi T, Clement JP 4th et al (1995) Reconstitution of IKATP: an inward rectifier subunit plus the sulfonylurea receptor. Science 270:1166-1170

14. Clement JP, Kunjilwar K, Gonzalez G, Schwanstecher M, Panten U, Aguilar-Bryan L, Bryan J (1997) Association and stoichiometry of K(ATP) channel subunits. Neuron 18:827-838

15. Aguilar-Bryan L, Nichols CG, Wechsler SW et al (1995) Cloning of the beta cell high-affinity sulfonylurea receptor: a regulator of insulin secretion. Science 268:423-426

16. Fan Z, Makielski JC (1997) Anionic phospholipids activate ATPsensitive potassium channels. J Biol Chem 272:5388-5395

17. Hellman B (1965) Studies in obese-hyperglycemic mice. Ann NY Acad Sci 131:541-558

18. Lacy PE, Kostianovsky M (1967) Method for the isolation of intact islets of Langerhans from the rat pancreas. Diabetes 16:35-39

19. Hamill OP, Marty A, Neher E, Sakmann B, Sigworth FJ (1981) Improved patch-clamp techniques for high-resolution current recording from cells and cell-free membrane patches. Pflugers Arch 391:85-100

20. Larsson O, Ämmälä C, Bokvist K, Fredholm B, Rorsman P (1993) Stimulation of the KATP channel by ADP and diazoxide requires nucleotide hydrolysis in mouse pancreatic beta-cells. J Physiol 463:349-365

21. Shrago E (2000) Long-chain acyl-CoA as a multi-effector ligand in cellular metabolism. J Nutr 130:290S-293S

22. Rapedius M, Soom M, Shumilina E et al (2005) Long chain CoA esters as competitive antagonists of phosphatidylinositol 4,5bisphosphate activation in Kir channels. J Biol Chem 280:30760-30767

23. Cukras CA, Jeliazkova I, Nichols CG (2002) Structural and functional determinants of conserved lipid interaction domains of inward rectifying Kir6.2 channels. J Gen Physiol 119:581-591

24. Tucker SJ, Gribble FM, Zhao C, Trapp S, Ashcroft FM (1997) Truncation of Kir6.2 produces ATP-sensitive $\mathrm{K}+$ channels in the absence of the sulphonylurea receptor. Nature 387:179-183

25. Tarasov AI, Girard CAJ, Ashcroft FM (2006) ATP sensitivity of the ATP-sensitive $\mathrm{K}+$ channel in intact and permeabilized pancreatic $\beta$-cells. Diabetes 55:2446-2454

26. Hisanaga Y, Ago H, Nakagawa N et al (2004) Structural basis of the substrate-specific two step catalysis of long chain fatty acidCoA synthetase dimer. J Biol Chem 279:31717-31726

27. Kuo A, Gulbis JM, Antcliff JF et al (2003) Crystal structure of the potassium channel KirBac1.1 in the closed state. Science 300:1922-1926

28. Schulze D, Rapedius M, Krauter T, Baukrowitz T (2003) Longchain acyl-CoA esters and phosphatidylinositol phosphates modulate ATP inhibition of KATP channels by the same mechanism. J Physiol 552:357-367

29. Shyng SL, Cukras CA, Harwood J, Nichols CG (2000) Structural determinants of PIP(2) regulation of inward rectifier K(ATP) channels. J Gen Physiol 116:599-608

30. John SA, Weiss JN, Ribalet B (2001) Regulation of cloned ATPsensitive $\mathrm{K}$ channels by adenine nucleotides and sulfonylureas: interactions between SUR1 and positively charged domains on Kir6.2. J Gen Physiol 118:391-405

31. Lin YW, Jia T, Weinsoft AM, Shyng SL (2003) Stabilization of the activity of ATP-sensitive potassium channels by ion pairs formed between adjacent Kir6.2 subunits. J Gen Physiol 122:225-237

32. Rohács T, Lopes CMB, Jin T, Ramdya PP, Molnár Z, Logothetis DE (2003) Specificity of activation by phosphoinositides determines lipid regulation of Kir channels. Proc Natl Acad Sci USA 100:745-750 